\title{
OsWRKY42 Represses OsMT1d and Induces Reactive Oxygen Species and Leaf Senescence in Rice
}

\author{
Muho Han ${ }^{1,2}$, Chi-Yeol Kim ${ }^{1,2}$, Junok Lee ${ }^{1}$, Sang-Kyu Lee ${ }^{1}$, and Jong-Seong Jeon ${ }^{1, *}$
}

\begin{abstract}
We isolated a rice (Oryza sativa L.) WRKY gene which is highly upregulated in senescent leaves, denoted OsWRKY42. Analysis of OsWRKY42-GFP expression and its effects on transcriptional activation in maize protoplasts suggested that the OsWRKY42 protein functions as a nuclear transcriptional repressor. OsWRKY42-overexpressing (OsWR $K Y 42 O X$ ) transgenic rice plants exhibited an early leaf senescence phenotype with accumulation of the reactive oxygen species (ROS) hydrogen peroxide and a reduced chlorophyll content. Expression analysis of ROS producing and scavenging genes revealed that the metallothionein genes clustered on chromosome 12, especially OsMT1d, were strongly repressed in OsWRKY42OX plants. An OsMT1d promoter:LUC construct was found to be repressed by OsWRKY42 overexpression in rice protoplasts. Finally, chromatin immunoprecipitation analysis demonstrated that OsWRKY42 binds to the W-box of the OsMT1d promoter. Our results thus suggest that OsWRKY42 represses OsMT1d-mediated ROS scavenging and thereby promotes leaf senescence in rice.
\end{abstract}

\section{INTRODUCTION}

Senescence is a final developmental process in the life cycle of plants and a type of programmed cell death. Senescence is controlled by multiple developmental factors and also external biotic and abiotic stress signals (Buchanan-Wollaston et al., 2005; Lim et al., 2007; Munne-Bosch and Alegre, 2004). Comprehensive analyses of altered gene expression patterns in genetically controlled senescent leaves, as a study model for plant senescence, has identified important regulatory factors. To date, over 100 transcription factors, most notably belonging

\footnotetext{
${ }^{1}$ Graduate School of Biotechnology and Crop Biotech Institute, Kyung Hee University, Yongin 446-701, Korea, ${ }^{2}$ These authors contributed equally to this work.

${ }^{*}$ Correspondence: jjeon @ khu.ac.kr
}

Received 15 May, 2014; revised 17 June, 2014; accepted 18 June, 2014; published online 31 July, 2014

Keywords: metallothionein, OsMT1d, OsWRKY42, reactive oxygen species, rice, senescence to the WRKY, NAC, C2H2 zinc finger, AP2/EREBP, and MYB families, have been identified to be highly upregulated in senescent leaves in Arabidopsis (Balazadeh et al., 2008; Buchanan-Wollaston et al., 2005; Guo et al., 2004). These results suggest that the transition from mature to senescent leaves is achieved by a massive reprogramming of gene expression.

The WRKY transcription factors constitute one of the largest transcription factor families in plants. WRKY proteins have the highly conserved DNA-binding domain of one or two 60 amino acid regions harboring WRKYGQK and a zinc finger structure. WRKYs have been shown to play important roles in the senescence process. For instance, the Arabidopsis atwrky53 mutant shows a delayed leaf senescence whereas AtWRKY53 overexpression resulted in precocious senescence, suggesting that AtWRKY53 functions as a positive regulator of leaf senescence (Miao et al., 2004; 2007). The Arabidopsis double mutant atwrky54/atwrky70 displays a significantly enhanced senescence phenotype, suggesting that AtWRKY70 and AtWRKY54 act as negative regulators of leaf senescence (Besseau et al., 2012).

The production of reactive oxygen species (ROS) is one of the earliest components of the leaf senescence pathway (Jing et al., 2008; Mittler et al., 2004; Zentgraf and Hemleben, 2008). For instance, AtWRKY53 and its regulators are controlled by hydrogen peroxide $\left(\mathrm{H}_{2} \mathrm{O}_{2}\right)$ (Miao et al., 2007; 2008). Cellular levels of ROS have been positively correlated with the severity of leaf senescence in Arabidopsis and oilseed rape (Bieker et al., 2012). The ROS status has been found to be controlled by a fine-tuned network of enzymatic and antioxidative components consisting of ROS-producing and ROS-scavenging proteins (Mittler et al., 2004; Kim et al., 2012). ROS are produced under stress conditions primarily as byproducts of normal metabolic processes, such as respiration and photosynthesis, in chloroplasts, mitochondria and peroxisomes and also at the cell surface and exterior by the activity of multiple enzymes including NADPH oxidases (Apel and Hirt, 2004; Mittler et al., 2004; Noctor et al., 2014). ROS are scavenged by enzymes such as superoxide dismutase, catalase, and peroxidase (Apel and Hirt, 2004; Jang et al., 2012) and also nonenzyme components that include low molecular antioxidants, such as ascorbate, glutathione, carotenoids, and metallothioneins (MTs) (Gechev et al., 2006). MTs harbor conserved cysteine-rich domains (Hassinen et al., 2011; Yang et al., 2009), and the OsMT1d and OsMT2b proteins in rice have also been shown to be ROS scavengers 
(Hu et al., 2011; Steffens and Sauter, 2009; Wong et al., 2004).

Few studies to date have focused on leaf senescence in rice, a vital commercial crop plant that feeds more than half of the world's population. In particular, the molecular regulatory mechanism of transcription factors underlying leaf senescence remains largely unknown in rice. The OsWRKY family comprises over 100 members in the rice genome (Rice WRKY Working Group, 2012). To date, most of the functionally characterized OsWRKYs have been reported to play roles in defense responses to biotic pathogens and also abiotic stress responses to environmental stimuli and hormones (De Vleesschauwer et al., 2013; Jang et al., 2010; Ryu et al., 2006). In our present study, we describe the isolation and characterization of a leaf senescence-inducible OsWRKY gene in rice, OsWRKY42. We provide evidence that the OsWRKY42 protein functions as a positive regulator of leaf senescence in rice by increasing the ROS level via the repression of the OsMT1d gene.

\section{MATERIALS AND METHODS}

\section{Plant materials and growth conditions}

Rice [japonica cultivar (cv.) Dongjin] plants were grown in a greenhouse under a $14 / 10 \mathrm{~h}$ light and dark period, at $24-28^{\circ} \mathrm{C}$ temperature and $70-80 \%$ humidity.

\section{RNA isolation and RT-PCR analysis}

Total RNA was prepared from various tissues of rice plants using Trizol reagent (Invitrogen, USA) with DNase treatment (TURBO DNA-free kit; Ambion-Life technologies, USA). The extracted RNA was reverse-transcribed using AMV reverse transcriptase XL (2620A; Takara, Japan) with RNase inhibitor (2312A; Takara) and an oligo-dT primer. The synthesized first strand CDNA was used in subsequent PCR reactions with gene-specific primers and control primers for OsUBQ5. The primers were designed in the region encompassing at least one intron for each gene to exclude any influence by genomic DNA contamination (Supplementary Table 1; Jain et al., 2006).

\section{Transient expression assay in mesophyll protoplasts}

To examine the transcriptional activation ability of OsWRKY42, the effector vector was constructed by fusing the OsWRKY42 cDNA amplified by PCR using FL-F/R primers containing Smal and Sall restriction sites, respectively (Supplementary Table 1). The resulting vector contained the $35 \mathrm{~S}$ promoter, the tobacco mosaic virus (TMV) translation enhancer $(\Omega)$ sequence, and the OsWRKY42 cDNA insert fused to the GAL4 DNA binding domain (BD) in frame. The OsWRKY30-carrying effector vector was then used as a control transcriptional activator (Han et al., 2013). The GAL4-responsive reporter vector contained $5 X$ GAL4, minimal TATA, the $\Omega$ sequence, and the Luciferase (LUC) gene (Ohta et al., 2000). The maize Ubiquitin1 promoter: $\beta$-glucuronidase (ZmUBQ1:GUS) construct was used as an internal control (Cho et al., 2009). Maize mesophyll protoplasts isolated from the second leaves of dark grown plants were cotransfected with the effector, reporter and internal control vectors as described previously (Cho et al., 2009).

To analyze transcriptional repression by OsWRKY42, two reporter vectors, OsMT1a:LUC and OsMT1d:LUC, were constructed with promoter regions from nucleotides -1496 to -1 and -1427 to -1 , respectively. These positions are relative to the +1 A translational initiation codons of OsMT1a and OsMT1d which were amplified by PCR using OsMT1a pro F/R and OsMT1d pro F/R primers containing $B a m H I$ and Sall restriction sites (Supplementary Table 1). The amplified fragments were in- serted into the JJ803 vector carrying LUC derived from pGL2 (Promega, USA). The 35S:OsWRKY42-cMyc fusion effector construct was generated by insertion of an OsWRKY42 cDNA fragment without the stop codon that had been amplified using GFP-F/R primers, between the $\Omega$ and c-Myc of the pJJ1754 vector (Supplementary Table 1). ZmUBQ1:GUS was used as an internal control (Cho et al., 2009). The constructed vectors were transformed into rice protoplasts isolated from the second leaves of young plants (Zhang et al., 2011) using the polyethylene glycol-calcium-mediated method (Cho et al., 2009). Transfected protoplasts were incubated for $12 \mathrm{~h}$ in W5 solution (Zhang et al., 2011) and harvested in lysis buffer (Promega). LUC activity was analyzed using a luciferase assay system (E1500; Promega) and a VICTOR2 multilabel counter (PerkinElmer, USA). GUS assays were performed using a previously described method (Jefferson et al., 1987). All transient expression experiments were repeated three times and gave similar results.

\section{Subcellular localization analysis of OsWRKY42}

The OsWRKY42 cDNA insert without its stop codon was amplified using GFP-F/R primers containing $X b a l$ and $X$ hol restriction sites, respectively (Supplementary Table 1). The amplified fragments were digested with the respective restriction enzymes and then subcloned between the $35 \mathrm{~S}$ promoter and sGFP of the pJJ461 vector derived from the binary vector pC1300intC (Ouwerkerk et al., 2001). The resulting GFP fusion construct was transiently expressed in maize protoplasts (Cho et al., 2009), which were subsequently examined by laserscanning confocal microscopy (LSM 510 META, Carl Zeiss, Germany). Chlorophyll autofluorescence (Han et al., 2012) and OsABF1-RFP (Han et al., 2013; Hossain et al., 2010; Truernit and Haseloff, 2008) were used as chloroplast and nuclear markers, respectively.

\section{Rice transformation}

OsWRKY42 cDNA was amplified by RT-PCR using the cDNA F/R primers containing Smal and Kpal restriction sites, respectively (Supplementary Table 1), and inserted between the ZmUBQ1 promoter and Nopaline synthase (Nos) terminator of pGA1611 (Kim et al., 2003). The resulting construct, $Z m U B Q 1$ : OsWRKY42, was used for rice (cv. Dongjin) transformation via Agrobacterium tumefaciens (LBA4404) as described previously (Jeon et al., 2000).

\section{Transient expression assay in tobacco leaves}

The 35S:OsWRKY42-cMyc, 35S:GFP (pJJ461) and the corresponding empty vector (pJJ1754) were introduced into $A$. tumefaciens strain GV3101. Overnight cultures were harvested and resuspended in $10 \mathrm{mM} \mathrm{MgCl} 2$ to an $\mathrm{OD}_{600}$ of 0.5 . After incubation at room temperature for $3 \mathrm{~h}$, the cells were used to infiltrate six-week-old Nicotiana benthamiana leaves using a needleless syringe $(1 \mathrm{ml})$ as described previously (Romeis et al., 2001; Seo et al., 2012). Plants were grown and maintained throughout the experiments in a growth chamber at a temperature of $25^{\circ} \mathrm{C}$ and under $12 \mathrm{~h}$ of light.

\section{Hydrogen peroxide assay}

For the detection of $\mathrm{H}_{2} \mathrm{O}_{2}$, fully expanded second leaves from the top of six-week-old wild type and transgenic plants were placed in solution of $1 \mathrm{mg} \mathrm{ml}^{-1} 3,3$-diaminobenzidine (DAB) and vacuum infiltrated at $650 \mathrm{mmHg}$ for $10 \mathrm{~min}$. The leaves were then immersed in DAB solution for $3 \mathrm{~h}$ in darkness. Subsequently, the treated leaves were decolorized in $80 \%$ ethanol, 
Regulatory Role of Rice OsWRKY42 in Leaf Senescence

Muho Han et al.

placed onto glass slides and observed under a stereomicroscope (Olympus, USA). The $\mathrm{H}_{2} \mathrm{O}_{2}$ content was also measured colorimetrically in the same fully expanded second leaves from the tops of six-week-old wild type and transgenic plants using $50 \mathrm{mM}$ phosphate buffer ( $\mathrm{pH}$ 6.5) containing $1 \mathrm{mM}$ hydroxylamine (Jana and Choudhuri, 1982). After centrifugation at 6,000 $\times g$ for $25 \mathrm{~min}$, the extracts were mixed with $0.1 \%$ titanium sulphate in $20 \%(\mathrm{v} / \mathrm{v}) \mathrm{H}_{2} \mathrm{SO}_{4}$. After centrifugation, the absorbance was measured at $410 \mathrm{~nm}$ and the $\mathrm{H}_{2} \mathrm{O}_{2}$ content was calculated using the extinction coefficient $0.28 \mu \mathrm{mol}^{-1} \mathrm{~cm}^{-1}$.

\section{Chlorophyll assay}

Rice leaves were prepared from the fully expanded second leaves from the tops of six-week-old wild type and transgenic plants. The total chlorophyll contents were measured spectrophotometrically after extraction in $80 \%$ acetone using the method of Porra et al. (1989).

\section{Promoter sequence analysis of OsMT1 genes}

The sequences at approximately $1.5-\mathrm{kb}$ upstream from the coding regions of OsMT1 genes were analyzed for the W-box using the PLACE Signal Scan program (Higo et al., 1999). Wbox elements harboring the consensus sequence were identified according to published criteria (Eulgem et al., 2000; Kim and Zhang, 2004).

\section{Chromatin immunoprecipitation analysis}

For chromatin immunoprecipitation (ChIP) analysis, the 35S: OsWRKY42-cMyc construct was transfected into rice protoplasts as described above. ChIP analysis was then performed using the EpiQuik Plant ChIP kit (Epigentek, USA) with the cMyc antibody (G3109; Santa Cruz Biotechnology, USA). Transfected protoplasts were harvested with $1 \%$ formaldehyde solution to cross-link the proteins to the DNA. The cross-linked DNA was sheared by sonication into 200-500 bp fragments in the presence of a protease inhibitor cocktail. A dilution of the resulting supernatant was incubated with an anti-cMyc- bound strip at room temperature for 90 min with orbital shaking. Relative enrichment was measured by comparing the input and ChIP values. Normal mouse IgG was used as a negative control antibody. All of the primers used in the ChIP assays are listed in Supplementary Table 1.

\section{RESULTS}

Identification of a senescence-inducible OsWRKY42 gene in rice leaves

Through a systematic screen of the digital expression profile of rice WRKY genes using the RiceXPro (Sato et al., 2011; http:// ricexpro.dna.affrc.go.jp/) database, we identified the OsWRK Y42 gene (LOC_Os02g26430) to be upregulated during the ripening stage that accompanies leaf senescence. Subsequent RT-PCR confirmed that OsWRKY42 is highly expressed only in senescent leaves, and is present at much lower levels in the young and mature leaves of rice plants (Fig. 1). The senescence-associated gene, Staygreen (SGR) (Park et al., 2007), was also found to be increased only in senescent leaves. The Rubisco small subunit $(R b c S)$ gene was strongly downregulated in the senescent rice leaves, while the housekeeping gene OsUBQ5 was constitutively transcribed. Our results thus indicated that OsWRKY42 is strongly upregulated in senescent rice leaves.

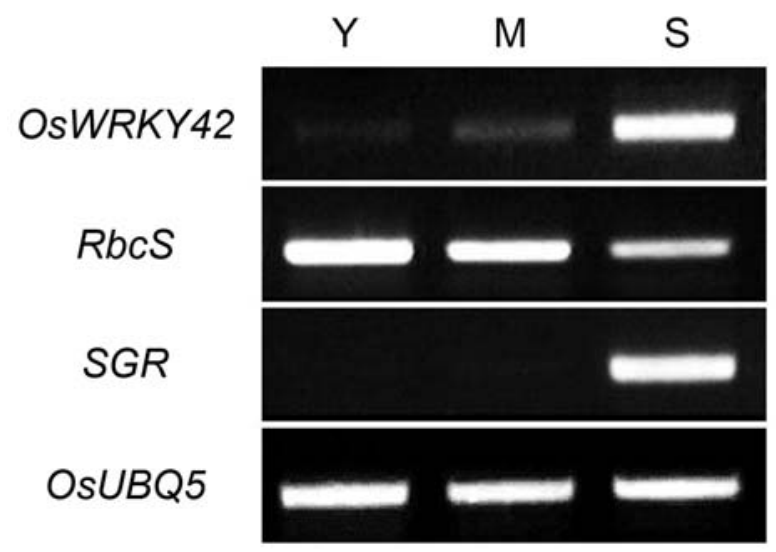

Fig. 1. Expression pattern of the OsWRKY42 gene in rice leaves. $R b c S$ was used as a senescence-downregulated marker, whilst $S G R$ was used as a senescence-inducible gene. $Y$, young leaves of three-week-old plants; M, fully expanded mature leaves of tenweek-old plants; $S$, senescent flag leaves of 17-week-old plants. OsUBQ5 was amplified as an internal RT-PCR control.

\section{Subcellular localization and transcriptional activator properties of OsWRKY42}

To next examine the subcellular localization of OsWRKY42, we generated an OsWRKY42-GFP fusion construct for introduction into maize mesophyll protoplasts (Fig. 2A). The green OsWRKY42-GFP signal merged very tightly with the RFP (red) signal of the OsABF1-RFP nuclear marker (Hossain et al., 2010). The auto-fluorescence of chlorophyll was used as a chloroplast marker. This indicated that the OsWRKY42 protein localizes in the nucleus.

The transcriptional activator ability of OsWRKY42 was examined using the maize protoplast transient expression system (Cho et al., 2009). The effector vectors used for this experiment contain either BD alone, or OsWRKY30 or OsWRKY42 fused to $\mathrm{BD}$ (Fig. 2B). BD- and BD-OsWRKY30 were used as negative and positive controls, respectively. In the maize protoplasts cotransfected with the effector and reporter vectors, the LUC activity of the effector BD-OsWRKY42 (OsW42) was reduced by about 0.4 -fold of the empty vector (EV) control (Fig. 2C). The known transcriptional activator OsWRKY30 (OsW30; Han et al., 2013) increased LUC reporter activity (Fig. 2C). This result suggests that OsWRKY42 functions as a transcriptional repressor in the nucleus.

\section{Phenotype of OsWRKY42 transgenic rice plants}

To further investigate the role of OsWRKY42 in rice, we produced 12 transgenic plants expressing ZmUBQ1:OsWRKY42. Ten of the primary transgenic rice plants that highly expressed OsWRKY42 displayed an extremely early onset of leaf senescence, eventually leading to plant death (Supplementary Fig. 1) Only two independent transgenic rice lines, OsWRKY42OX1 and OsWRKY42OX3, with relatively low transgene expression could grow and set a small number of seeds. The progeny of these plants were analyzed in further experiments (Fig. 3A) and clearly showed an early leaf senescence phenotype at the three-week-old plant stage (Fig. 3B).

To examine whether OsWRKY42 could induce early leaf senescence in other heterologous species, we expressed the gene under the control of $35 \mathrm{~S}$ promoter in tobacco leaves by 
A

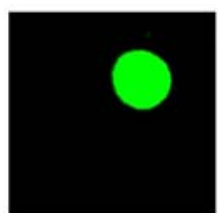

OsWRKY42GFP

B

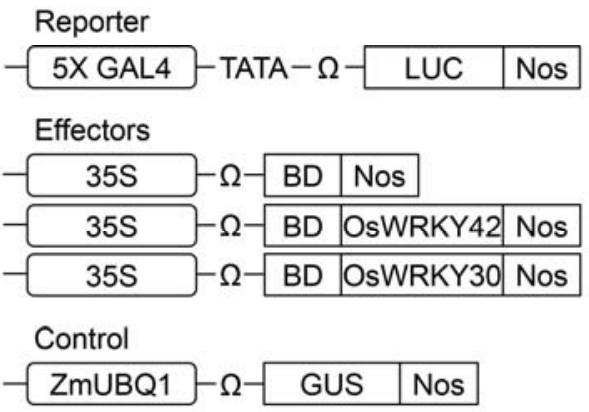

A
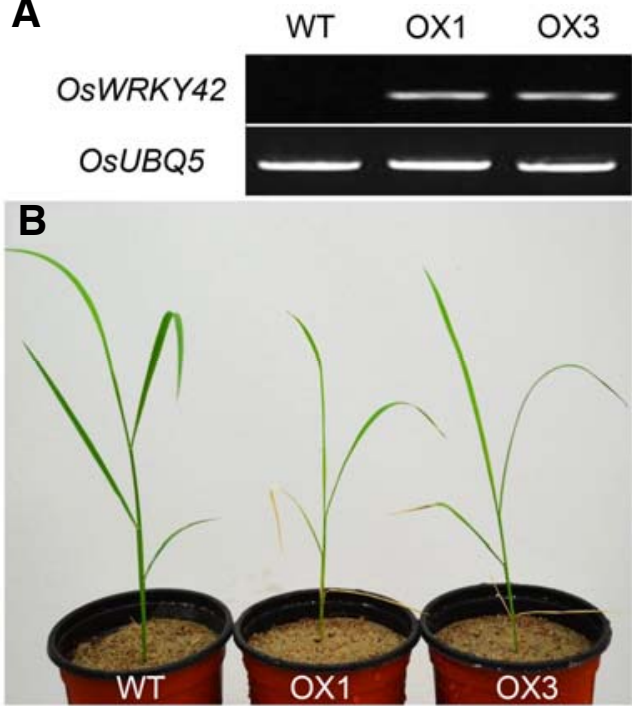

Fig. 3. Characterization of OsWRKY42-overexpressing (OsWRKY42OX) transgenic rice plants. (A) RT-PCR analysis of OsWRKY42 expression in OsWRKY $42 O X 1$ (OX1) and OsWRKY42OX3 (OX3) transgenic lines. OsUBQ5 was amplified as an internal RT-PCR control. (B) Phenotype of three-week-old OsWRKY42OX transgenic lines (OX1 and OX3) in comparison with wild type (WT).
Agrobacterium-mediation (Supplementary Fig. 2) (Romeis et al., 2001; Seo et al., 2012). At 10 days after inoculation, only Os WRKY42-cMyc expression (OsW42) caused early leaf senescence compared to the controls, Agrobacterium background GV3101 strain and Agrobacterium carrying empty vector (EV) or 35S:GFP (GFP). This result suggests that OsWRKY42 can also function as a positive regulator of leaf senescence in plant species other than rice.

\section{Hydrogen peroxide measurements in OsWRKY42} transgenic rice plants

A disruption of the critical and tightly regulated balance between the production and scavenging of ROS has been well established as a promoter of leaf senescence (Jing et al., 2008;
Mittler et al., 2004; Zentgraf and Hemleben, 2008). The early leaf senescence phenotype we observed in our current analysis prompted us examine whether the ROS level was increased in OsWRKY42OX transgenic rice lines. We thus examined the level of $\mathrm{H}_{2} \mathrm{O}_{2}$, the most significant ROS with a relatively long half-life, in the fully expanded second leaves from top of sixweek-old OsWRKY42OX1 and OsWRKY42OX3 lines. DAB staining exhibited strong brown colors in the leaves of both transgenic lines compared to wild type (Fig. 4A). Consistently, we found increased levels of $\mathrm{H}_{2} \mathrm{O}_{2}$ in the leaves of OsWRKY$42 O X 1$ and OsWRKY42OX3 lines (Fig. 4B), which also showed lower total chlorophyll contents than wild type plants (Fig. 4C). These results suggest that OsWRKY42 overexpression induces ROS accumulation and promotes leaf senescence. 


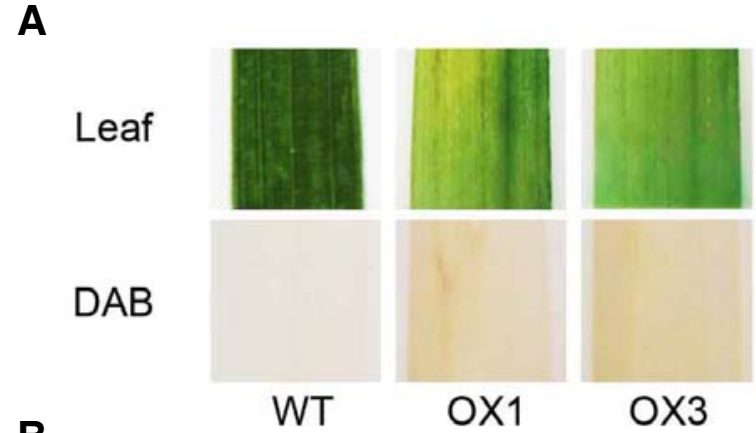

B
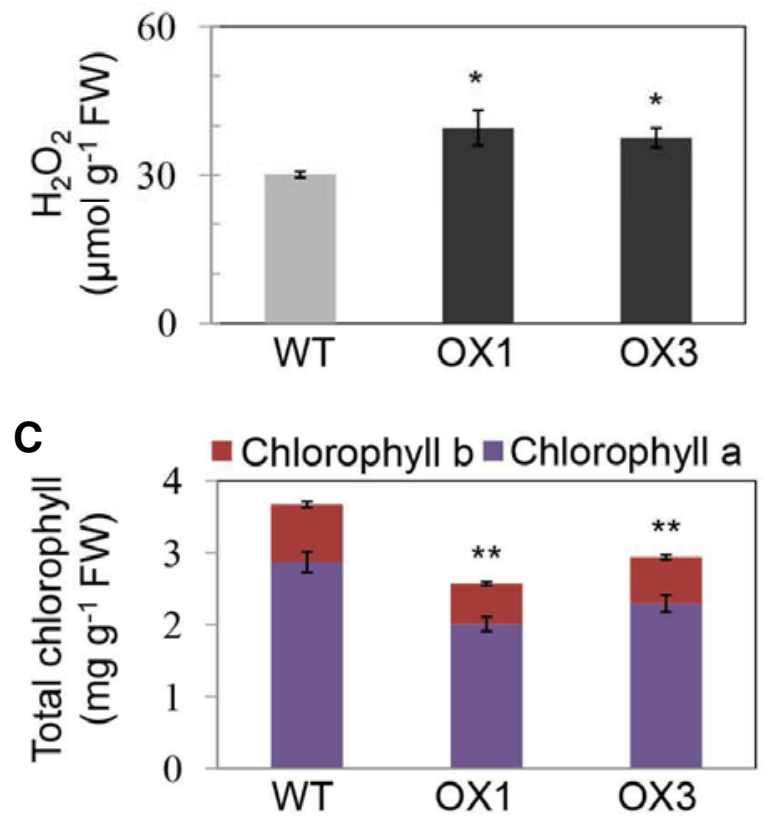

Fig. 4. $D A B$ staining for measurement of the relative $\mathrm{H}_{2} \mathrm{O}_{2}$ levels and total chlorophyll contents in the fully expanded second leaves from the top of six-week-old OsWRKY42OX1 (OX1) and OsWRK Y42OX3 (OX3) transgenic plants and wild type rice plants. (A) Representative leaves visualized by DAB staining of OsWRKY42OX lines and wild type (WT). (B) $\mathrm{H}_{2} \mathrm{O}_{2}$ levels of the OsWRKY42OX lines and wild type (WT). (C) Histogram of total chlorophyll contents of OsWRKY42OX lines and wild type (WT). Each datapoint represents the mean $( \pm \mathrm{SD})$ from three separate experiments. ${ }^{*} P<0.05$, ${ }^{* *} P<0.01$.

\section{Expression of ROS producing and scavenging genes in} OsWRKY42 transgenic rice plants

To examine whether any ROS producing and scavenging genes were disrupted in OsWRKY42OX1 and OsWRKY42OX3 lines, we compared the expression levels of a number of previously described ROS-related genes (Hu et al., 2011) in transgenic lines and wild type plants. Interestingly, we found that the highly expressed gene OsMT1d was strongly downregulated in OsWRKY42OX lines compared to wild type. The weakly expressed genes, OsMT1f and OsMT1g, were also found to be repressed in OsWRKY42OX lines (Fig. 5). In contrast, none of the other tested ROS producing and scavenging genes were significantly altered in this comparison (Supplementary Fig. 3). This indicates that the overexpression of OsWRKY42 re-

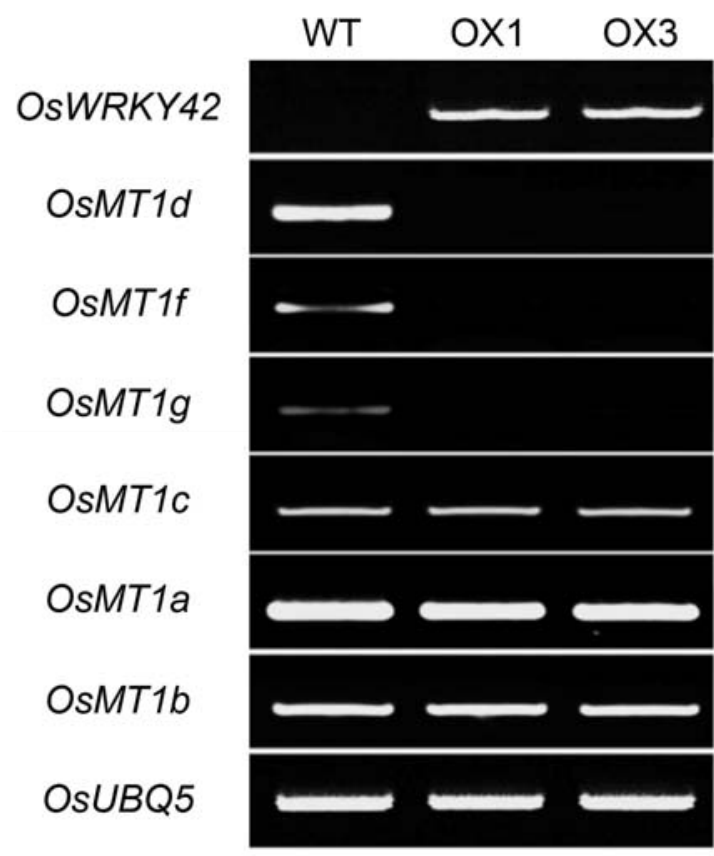

Fig. 5. Expression analysis of rice metallothionein I (OsMT1) genes in the leaves of OsWRKY42OX1 (OX1) and OsWRKY42OX3 (OX3) transgenic rice lines. Locus numbers of OsMT1 genes are: OsMT1a, LOC_Os12g38270; OsMT1b, LOC_Os03g17870; OsMT1c, LOC_Os12g38051; OsMT1d, LOC_Os12g38300; OsMT1f, LOC_ Os12g38010; OsMT1g, LOC_Os12g38290. OsUBQ5 transcripts were amplified as an internal RT-PCR control.

presses ROS non-enzyme scavenger MT1 genes, in particular OsMT1d clustered in a short interval of chromosome 12, and thereby induces ROS accumulation and precocious leaf senescence.

\section{Analysis of OsMT1d transcriptional repression by} OsWRKY42 in rice protoplasts

Analysis of about $1.5 \mathrm{~kb}$ upstream regulatory sequences of OsMT1d, OsMT1f and OsMT1g, revealed that these three genes each harbor three potential W-boxes, which are known as WRKY binding elements (Supplementary Fig. 4; Eulgem et al., 2000; Kim and Zhang, 2004). To examine whether OsWRKY42 represses OsMT1 genes by binding to these Wboxes, we performed a transient expression assay using LUC reporter system (Fig. 6). For this experiment, we isolated promoters of OsMT1d (the nucleotides -1427 -1) and OsMT1a (1496 -1) as positive (the most strongly repressed OsMT1 gene in OsWRKY42OX lines) and negative (constantly expressed OsMT1 gene) downstream targets, respectively (Fig. 6A). After coexpression of reporter and effector vectors in rice protoplasts, we found that the expression of OsWRKY42-cMyc strongly repressed OsMT1d:LUC expression but not OsMT1a:LUC (Fig. $6 \mathrm{~B})$. This indicates that OsWRKY42 represses OsMT1d expression.

Analysis of promoter binding by OsWRKY42 in vivo The OsMT1d promoter contains three potential W-boxes, two copies (-1099 -1094 and -945 -940 within P2) that harbor a consensus sequence variant A/CTGACC and one copy (-729 - 
A

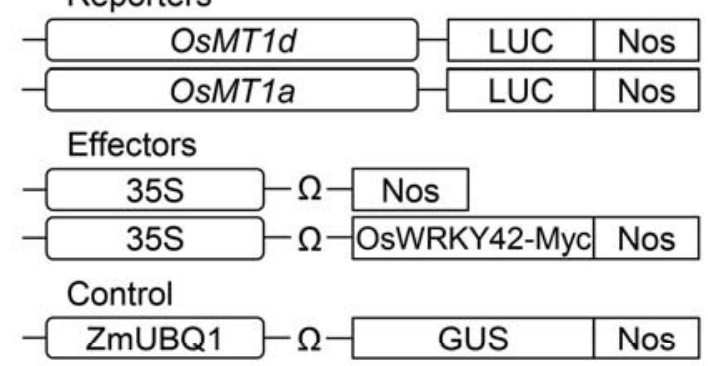

B

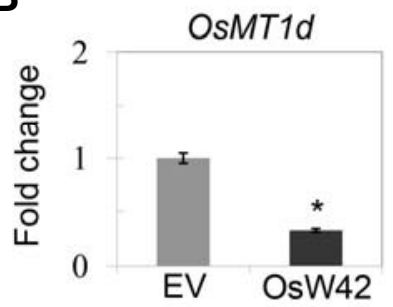

C
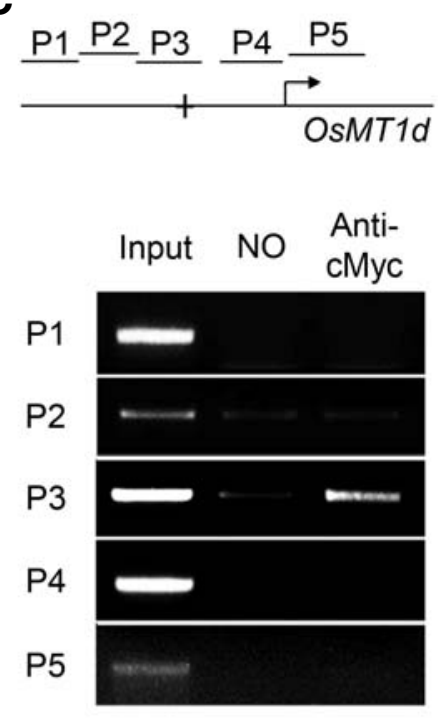

Fig. 6. Transient expression analysis of promoters of OsMT1a and OsMT1d and ChIP analysis of the OsMT1d promoter in rice protoplasts. (A) Schematic representation of reporters and effectors. OsMT1a and OsMT1d promoters contain the nucleotides -1496 to -1 and -1427 to -1 , respectively. $Z m U B Q 1$ : GUS was used an internal control. (B) Fold changes calculated using a luciferase assay for the changed expression level of target promoter:LUC constructs by expression of 35S:OsWRKY42-cMyc (OsW42) in rice protoplasts. LUC activities were normalized after transfection with the empty vector (EV) control that was arbitrarily assigned a value of $1 .{ }^{\star} P<0.01$. (C) Selected promoter fragments of OsMT1d (top). ChIP analysis was performed of the OsMT1d promoter using chroma-

tin prepared from 35S:OSWRKY42-cMyc transfected rice protoplasts (bottom). + , W-box with the consensus sequence TTGACT.

724 within P3) with the consensus sequence TTGACT (Fig. 6C, top; Supplementary Fig. 4). To verify the in vivo association of OsWRKY42 and the W-box present in the OsMT1d promoter, we performed chromatin immunoprecipitation (ChIP) analysis (Fig. 6C, bottom). We analyzed DNA before immunoprecipitation (Input) and immunoprecipitated DNA by PCR using primers that amplify the P1 to P5 regions of the OsMT1d promoter. The ChIP assay revealed that the OsWRKY42-cMyc protein specifically binds to the P3 region on the OsMT1d promoter (Fig. 6C, bottom). This result indicates that the OsWRKY42 transcriptional repressor binds to the W-box region of the OsMT1d promoter and represses its expression. This in turn disrupts the ROS balance which results in the precocious leaf senescence seen in OsWRKY42OX transgenic rice plants.

\section{DISCUSSION}

The processes underlying senescence influence many important agricultural traits including the number and quality of seeds and timing of seed setting. However, our knowledge concerning the regulatory mechanisms that control senescence is still limited in rice, one of the world's most agronomically important crops. It has been evident that ROS trigger leaf senescence (Zentgraf and Hemleben, 2008). Plants have developed a finetuned network of enzymatic and low-molecular-weight antioxidative components to regulate their ROS status. Oxidative stress and senescence occur when this critical balance is disrupted because of a depletion of antioxidants or excess accumulation of ROS.

The association between ROS and the WRKY transcription factors was revealed by the finding that the Arabidopsis AtWRKY53 gene is induced by $\mathrm{H}_{2} \mathrm{O}_{2}$ treatment (Miao et al., 2004). $\mathrm{H}_{2} \mathrm{O}_{2}$ was found to induce reporter gene expression driven by the AtWRKY53 promoter in protoplasts prepared from wild type plants. In contrast, this induction was abolished in protoplasts prepared from AtWRKY53 knockout lines, indicating that the AtWRKY53 protein is directly involved in the positive regulation of genes that respond to $\mathrm{H}_{2} \mathrm{O}_{2}$ (Miao et al., 2007).
AtWRKY70 was also found previously to be constitutively expressed in an ROS scavenging enzyme gene mutant, atapx1, revealing its responsiveness to ROS (Ciftci-Yilmaz et al., 2007). However, it remains to be determined how these WRKY factors can participate in ROS homeostasis in the senescent leaf. In our current study, we found that the rice OsWRKY42 transcription factor functions as a key repressor of antioxidant OsMT1 isoforms, in particular MT1d. Our present findings thus provide direct evidence that WRKY can directly regulate ROS scavenging genes during the onset of leaf senescence.

ROS participate in a diverse range of plant processes including pollen development (Hiscock and Allen, 2008; Mclnnis et al., 2006a; 2006b). In this regard, it is noteworthy that the OsMT1d (former name MT-1-4b) gene has been previously shown to be a downstream target of the OSMADS3 gene that regulates late anther development and pollen formation in rice ( $\mathrm{Hu}$ et al., 2011). Microarray analysis of an OsMADS3 mutant allele, mads3-4, which displays defective anther walls, aborted microspores, and complete male sterility, revealed the altered expression of a number of ROS producing and scavenging genes in this mutant. OsMADS3 protein was further found to bind to the consensus CArG- core element [CC(A/T)4NNGG] of promoter of OsMT1d. In addition, a recombinant OsMT1d revealed ROS scavenging activity in a previous study (Hu et al., 2011). Thus, the modulation of the ROS level through OsMT1d via OsMADS3 appears to be essential for rice male reproductive development. Taken together with previous findings, our current study results suggest that at least two transcription factors, OsMADS3 and OsWRKY42 regulate OsMT1d positively and negatively, respectively, to balance the ROS levels. It would be interesting in the future to examine whether OsWRKY42 has any function in rice male development.

Histochemical GUS analysis of a serial deletion of the rice OsMT1d promoter in transgenic Arabidopsis revealed an important promoter region demarcated by nucleotides -1052 -583, a region which contains metal-responsive cis-elements such as a putative animal metal-regulatory motif and a potential copper responsive element which responds to treatments with different 
heavy metals, such as $\mathrm{Cu}^{2^{+}}, \mathrm{Pb}^{2^{+}}$and $\mathrm{Al}^{3^{+}}$(Dong et al., 2010). It would be therefore interesting to determine whether the $\mathrm{W}$ box element at -729 -724, defined as the OsWRKY42 binding site in our present study and located in the interval of the important OsMT1d promoter region, can also participate in the regulatory response to heavy metals.

The OsMT1a, OsMT1c, OsMT1d, OsMT1f and OsMT1g genes are tightly clustered on chromosome 12. In RT-PCR analysis of the shoots of rice seedlings, three of these genes, OsMT1a, OsMT1c and OsMT1d, with high transcript levels were downregulated under salinity stress and ABA treatment, whilst they were previously found to be upregulated after drought stress (Kumar et al., 2012). This result suggests that these clustered OsMT1 genes may be coregulated under certain stress conditions. Consistently, we found in our current experiments that OsMT1d, OsMT1f, and OsMT1g are downregulated in OsWRKY42 transgenic rice plants, although OsMT1a, OsMT1b, and OsMT1c showed unaltered expression (Fig. 5). In our current study also, we confirmed that OsWRKY42 can bind to the OsMT1d promoter (Fig. 6). Given that the promoters of OsMT1f and OsMT1g, as well as of OsMT1d, contain conserved Wboxes (Supplementary Fig. S4) and both are similarly downregulated in OsWRKY42OX lines, it is likely that OsWRKY42 also associates with the regulatory elements of OsMT1f and OsMT1g genes and represses both genes.

In summary, we have here isolated and characterized a rice leaf senescence-inducible factor, OsWRKY42. Analysis of transgenic rice plants overexpressing OsWRKY42 has revealed a novel regulatory mechanism by which WRKY can participate in ROS balancing and in the modulation of leaf senescence. To achieve a more in-depth understanding of OsWRKY42 function during leaf senescence, it would be valuable in the future to identify additional targets of this gene through genome wide expression analysis of OsWRKY42OX transgenic rice.

Note: Supplementary information is available on the Molecules and Cells website (www.molcells.org).

\section{ACKNOWLEDGMENTS}

We thank Dr. Hideaki Shinshi (National Institute of Advanced Industrial Science and Technology, Japan) for providing the GAL4-responsive vector system and Dr. Shinyoung Lee (Academy of New Biology for Plant Senescence and Life History, DGIST) for helpful suggestion. This work was supported by grants from the Next-Generation BioGreen 21 Program, Rural Development Administration (PJ008156012013), and from the Mid-Career Researcher Program, National Research Foundation (NRF-2013R1A2A2A01068887).

\section{REFERENCES}

Apel, K., and Hirt, H. (2004). Reactive oxygen species: metabolism, oxidative stress, and signal transduction. Annu. Rev. Plant. Biol. 55, 373-399.

Balazadeh, S., Riaño-Pachón, D.M., and Mueller-Roeber, B. (2008) Transcription factors regulating leaf senescence in Arabidopsis thaliana. Plant Biol. 10, 63-75.

Bieker, S., Riester, L., Stahl, M., Franzaring, J., and Zentgraf, U. (2012). Senescence-specific alteration of hydrogen peroxide levels in Arabidopsis thaliana and oilseed rape spring variety Brassica napus L. cv. Mozart. J. Integr. Plant Biol. 54, 540-554.

Besseau, S., Li, J., and Palva, E.T. (2012). WRKY54 and WRKY70 co-operate as negative regulators of leaf senescence in Arabidopsis thaliana. J. Exp. Bot. 63, 2667-2679.

Buchanan-Wollaston, V., Page, T., Harrison, E., Breeze, E., Lim, P.O., Nam, H.G., Lin, J.F., Wu, S.H., Swidzinski, J., Ishizaki, K., et al. (2005). Comparative transcriptome analysis reveals signifi- cant differences in gene expression and signaling pathways between developmental and dark/starvation-induced senescence in Arabidopsis. Plant J. 42, 567-585.

Cho, J.I., Ryoo, N., Eom, J.S., Lee, D.W., Kim, H.B., Jeong, S.W., Lee, Y.H., Kwon, Y.K., Cho, M.H., Bhoo, S.H., et al. (2009). Role of the rice hexokinases OsHXK5 and OsHXK6 as glucose sensors. Plant Physiol. 149, 745-759.

Ciftci-Yilmaz, S., Morsy, M.R., Song, L., Coutu, A., Krizek, B.A., Lewis, M.W., Warren, D., Cushman, J., Connolly, E.L., and Mittler, R. (2007). The EAR-motif of the Cys2/His2-type zinc finger protein Zat7 plays a key role in the defense response of Arabidopsis to salinity stress. J. Biol. Chem. 282, 9260-9268.

De Vleesschauwer, D., Gheysen, G., and Höfte, M. (2013). Hormone defense networking in rice: tales from a different world. Trends Plant Sci. 18, 555-565.

Dong, C.J., Wang, Y., Yu, S.S., and Liu, J.Y. (2010). Characterization of a novel rice metallothionein gene promoter: its tissue specificity and heavy metal responsiveness. J. Integr. Plant Biol. 52, 914-924.

Eulgem, T., Rushton, P.J., Robatzek, S., and Somssich, I.E. (2000). The WRKY superfamily of plant transcription factors. Trends Plant Sci. 5, 199-206.

Gechev, T.S., Van Breusegem, F., Stone, J.M., Denev, I., and Laloi, C. (2006). Reactive oxygen species as signals that modulate plant stress responses and programmed cell death. Bioessays 28, 1091-1101.

Guo, Y., Cai, Z., and Gan, S. (2004). Transcriptome of Arabidopsis leaf senescence. Plant Cell Environ. 27, 521-549.

Han, S.H., Sakuraba, Y., Koh, H.J., and Paek, N.C. (2012). Leaf variegation in the rice zebra2 mutant is caused by photoperiodic accumulation of tetra-cis-lycopene and singlet oxygen. Mol. Cells 33, 87-97.

Han, M., Ryu, H.S., Kim, C.Y., Park, D.S., Ahn, Y.K., and Jeon, J.S. (2013). OsWRKY3O is a transcription activator that enhances rice resistance to the Xanthomonas oryzae pathovar oryzae. J. Plant Biol. 56, 258-265.

Hassinen, V.H., Tervahauta, A.I., Schat, H., and Karenlampi, S.O. (2011). Plant metallothioneins--metal chelators with ROS scavenging activity? Plant Biol. 13, 225-232.

Higo, K., Ugawa, Y., Iwamoto, M., and Korenaga, T. (1999). Plant cis-acting regulatory DNA elements (PLACE) database: 1999. Nucleic Acids Res. 27, 297-300.

Hiscock, S.J., and Allen, A.M. (2008). Diverse cell signaling pathways regulate pollen-stigma interactions: the search for consensus. New Phytol. 179, 286-317.

Hossain, M.A., Cho, J.I., Han, M., Ahn, C.H., Jeon, J.S., An, G., and Park, P.B. (2010). The ABRE-binding bZIP transcription factor OsABF2 is a positive regulator of abiotic stress and ABA signaling in rice. J. Plant Physiol. 167, 1512-1520.

Hu, L., Liang, W., Yin, C., Cui, X., Zong, J., Wang, X., Hu, J., and Zhang, D. (2011). Rice MADS3 regulates ROS homeostasis during late anther development. Plant Cell 23, 515-533.

Jain, M., Tyagi, A.K., and Khurana, J.P. (2006). Overexpression of putative topoisomerase 6 genes from rice confers stress tolerance in transgenic Arabidopsis plants. FEBS J. 273, 5245-5260.

Jana, S., and Choudhuri, M.A. (1982). Changes in the activities of ribulose 1,5-bisphosphate and phosphoenolpyruvate carboxylases in submersed aquatic angiosperms during aging. Plant Physiol. 70, 1125-1127.

Jang, J.Y., Choi, C.H., and Hwang, D.J. (2010). The WRKY superfamily of rice transcription factors. Plant Pathol. J. 26, 110-114.

Jang, S.J., Wi S.J., Choi, Y.J., An, G., and Park, K.Y. (2012). Increased polyamine biosynthesis enhances stress tolerance by preventing the accumulation of reactive oxygen species: T-DNA mutational analysis of Oryza sativa lysine decarboxylase-like protein 1. Mol. Cells 34, 251-262.

Jefferson, R.A., Kavanagh, T.A., and Bevan, M.W. (1987). GUS fusions: beta-glucuronidase as a sensitive and versatile gene fusion marker in higher plants. EMBO J. 6, 3901-3907.

Jeon, J.S., Lee, S., Jung, K.H., Jun, S.H., Jeong, D.H., Lee, J., Kim, C., Jang, S., Yang, K., Nam, J., et al. (2000). T-DNA insertional mutagenesis for functional genomics in rice. Plant J. 22, 561570.

Jing, H.C., Hebeler, R., Oeljeklaus, S., Sitek, B., Stuhler, K., Meyer, H.E., Sturre, M.J., Hille, J., Warscheid, B., and Dijkwel, P.P (2008). Early leaf senescence is associated with an altered cel- 
lular redox balance in Arabidopsis cpr5/old1 mutants. Plant Biol. 1, 85-98.

Kim, C.Y., and Zhang, S. (2004). Activation of a mitogen-activated protein kinase cascade induces WRKY family of transcription factors and defense genes in tobacco. Plant J. 38,142-151.

Kim, S.R., Lee, S., Kang, H.G., Jeon, J.S., Kim, K.M., and An, G. (2003). A complete sequence of the pGA1611 binary vector. J. Plant Biol. 46, 211-214.

Kim, I.S., Kim, Y.S., and Yoon, H.S. (2012). Rice ASR1 protein with reactive oxygen species scavenging and chaperone-like activities enhances acquired tolerance to abiotic stresses in Saccharomyces cerevisiae. Mol. Cells 33, 285-293.

Kumar, G., Kushwaha, H.R., Panjabi-Sabharwal, V., Kumari, S., Joshi, R., Karan, R., Mittal, S., Pareek, S.L., and Pareek, A. (2012) Clustered metallothionein genes are co-regulated in rice and ectopic expression of OsMT1e-P confers multiple abiotic stress tolerance in tobacco via ROS scavenging. BMC Plant Biol. 12, 107.

Lim, P.O., Kim, H.J., and Nam, H.G. (2007). Leaf senescence. Annu. Rev. Plant Biol. 58, 115-136.

Miao, Y., Laun, T., Zimmermann, P., and Zentgraf, U. (2004). Targets of the WRKY53 transcription factor and its role during leaf senescence in Arabidopsis. Plant Mol. Biol. 55, 853-867.

Miao, Y., Laun, T.M., Smykowski, A., and Zentgraf, U. (2007). Arabidopsis MEKK1 can take a short cut: it can directly interact with senescence-related WRKY53 transcription factor on the protein level and can bind to its promoter. Plant Mol. Biol. 65, 63-76.

Miao, Y., Smykowski, A., and Zentgraf, U. (2008). A novel upstream regulator of WRKY53 transcription during leaf senescence in Arabidopsis thaliana. Plant. Biol. 1, 110-120.

Mittler, R., Vanderauwera, S., Gollery, M., and Van Breusegem, F. (2004). Reactive oxygen gene network of plants. Trends Plant Sci. 9, 490-498.

McInnis, S.M., Desikan, R., Hancock, J.T., and Hiscock, S.J. (2006a). Production of reactive oxygen species and reactive nitrogen species by angiosperm stigmas and pollen: potential signaling crosstalk? New Phytol. 172, 221-228.

McInnis, S.M., Emery, D.C., Porter, R., Desikan, R., Hancock, J.T., and Hiscock, S.J. (2006b). The role of stigma peroxidases in flowering plants: insights from further characterization of a stigma-specific peroxidase (SSP) from Senecio squalidus (Asteraceae). J. Exp. Bot. 57, 1835-1846

Munne-Bosch, S., and Alegre, L. (2004). Die and let live: leaf senescence contributes to plant survival under drought stress. Funct. Plant Biol. 31, 203-216.

Noctor, G., Mhamdi, A., and Foyer, C.H. (2014). The roles of reactive oxygen metabolism in drought: not so cut and dried. Plant Physiol. 164, 1636-1648.

Ohta, M., Ohme-Takagi, M., and Shinshi, H. (2000). Three ethylene-responsive transcription factors in tobacco with distinct transactivation functions. Plant J. 1, 29-38

Ouwerkerk, P.B., de Kam, R.J., Hoge, J.H., and Meijer, A.H. (2001). Glucocorticoid-inducible gene expression in rice. Planta 213,
370-378

Park, S.Y., Yu, J.W., Park, J.S., Li, J., Yoo, S.C., Lee, N.Y., Lee, S.K., Jeong, S.W., Seo, H.S., Koh, H.J., et al. (2007). The senescence-induced Staygreen protein regulates chlorophyll degradation. Plant Cell 19, 1649-1664.

Porra, R.J., Thompson, W.A., and Kriedemann, P.E. (1989). Determination of accurate extinction coefficients and simultaneous equations for assaying chlorophylls $a$ and $b$ extracted with four different solvents: verification of the concentration of chlorophyl standards by atomic absorption spectrometry. Biochim. Biophys. Acta 975, 384-394.

Rice WRKY Working Group (2012). Nomenclature report on rice WRKY's - Conflict regarding gene names and its solution. Rice $5,1-3$.

Romeis, T., Ludwig, A.A., Martin, R., and Jones, J.D. (2001). Calcium-dependent protein kinases play an essential role in a plant defense response. EMBO J. 20, 5556-5567.

Ryu, H.S., Han, M., Lee, S.K., Cho, J.I., Ryoo, N., Heu, S., Lee, Y.H., Bhoo, S.H., Wang, G.L., Hahn, T.R., et al. (2006). A comprehensive expression analysis of the WRKY gene superfamily in rice plants during defense response. Plant Cell Rep. 25, 836847.

Sato, Y., Antonio, B.A., Namiki, N., Takehisa, H., Minami, H., Kamatsuki, K., Sugimoto, K., Shimizu, Y., Hirochika, $\mathrm{H}$., and Nagamura, Y. (2011). RiceXPro: a platform for monitoring gene expression in japonica rice grown under natural field conditions. Nucleic Acids Res. 39, D1141-D1148.

Seo, E., Yeom, S.I., Jo, S., Jeong, H., Kang, B.C., and Choi, D. (2012). Ectopic expression of Capsicum-specific cell wall protein Capsicum annuum senescence-delaying 1 (CaSD1) delays senescence and induces trichome formation in Nicotiana benthamiana. Mol. Cells 33, 415-422.

Steffens, B., and Sauter, M. (2009). Heterotrimeric G protein signaling is required for epidermal cell death in rice. Plant Physiol. 151 732-740.

Truernit, E., and Haseloff, J. (2008). A simple way to identify nonviable cells within living plant tissue using confocal microscopy Plant Methods 4, 15

Wong, H.L., Sakamoto, T., Kawasaki, T., Umemura, K., and Shimamoto, K. (2004). Down-regulation of metallothionein, a reactive oxygen scavenger, by the small GTPase OsRac1 in rice. Plant Physiol. 135, 1447-1456.

Yang, Z., Wu, Y., Li, Y., Ling, H.Q., and Chu, C. (2009). OsMT1a, a type 1 metallothionein, plays the pivotal role in zinc homeostasis and drought tolerance in rice. Plant Mol. Biol. 70, 219-229.

Zentgraf, U., and Hemleben, V. (2008). Molecular cell biology: are reactive oxygen species regulators of leaf senescence? Progress Bot. 69, 117-138.

Zhang, Y., Su, J., Duan, S., Ao, Y., Dai, J., Liu, J., Wang, P., Li, Y., Liu, B., Feng, D., et al. (2011). A highly efficient rice green tissue protoplast system for transient gene expression and studying light/chloroplast-related processes. Plant Methods 7, 30. 